\title{
Association between Exposure to Ambient Air Particulates and Metabolic Syndrome Components in a Saudi Arabian Population
}

\author{
Magdy Shamy ${ }^{1, *}$, Mansour Alghamdi ${ }^{1}$, Mamdouh I. Khoder ${ }^{1,2}{ }^{,}$Abdullah M. Mohorjy ${ }^{3}$, \\ Alser A. Alkhatim ${ }^{1}$, Abdulrahman K. Alkhalaf ${ }^{4}$, Jason Brocato ${ }^{5}$, Lung Chi Chen ${ }^{5}$, \\ George D. Thurston ${ }^{5}$, Chris C. Lim ${ }^{5}$ and Max Costa ${ }^{5}$ \\ 1 Department of Environmental Sciences, Faculty of Meteorology, Environment and Arid Land Agriculture, \\ King Abdulaziz University, Jeddah 21589, Saudi Arabia; mans99@gmail.com (M.A.); \\ khoder_55@yahoo.com (M.I.K.); abuenas@hotmail.com (A.A.A.) \\ 2 Center of Excellence in Environmental Studies, King Abdulaziz University, Jeddah 21589, Saudi Arabia \\ 3 Department of Civil Engineering, Faculty of Engineering, King Abdulaziz University, \\ Jeddah 21589, Saudi Arabia; amohorjy@kau.edu.sa \\ 4 Department of Meteorology, Faculty of Meteorology, Environment and Arid Land Agriculture, \\ King Abdulaziz University, Jeddah 21589, Saudi Arabia; akhalaf@kau.edu.sa \\ 5 Department of Environmental Medicine, New York University School of Medicine, \\ New York, NY 10987, USA; jab824@nyu.edu (J.B.); lung-chi.chen@nyumc.org (L.C.C.); \\ george.thurston@nyumc.org (G.D.T.); cchaeha@gmail.com (C.C.L.); max.costa@nyumc.org (M.C.) \\ * Correspondence: mshamy@kau.edu.sa; Tel.: +966-53-227-1120
}

Received: 3 December 2017; Accepted: 19 December 2017; Published: 25 December 2017

\begin{abstract}
Recent epidemiological evidence suggests that exposure to particulates may be a factor in the etiology of metabolic syndrome (MetS). In this novel study, we investigated the relationship between particulate levels and prevalence of MetS component abnormalities (hypertension, hyperglycemia, obesity) in a recruited cohort $(\mathrm{N}=2025)$ in Jeddah, Saudi Arabia. We observed significant associations between a $10 \mu \mathrm{g} / \mathrm{m}^{3}$ increase in $\mathrm{PM}_{2.5}$ and increased risks for MetS (Risk Ratio (RR): 1.12; 95\% Confidence Interval (CI): 1.06-1.19), hyperglycemia (RR: 1.08; 95\% CI: 1.03-1.14), and hypertension (RR: 1.09; 95\% CI: 1.04-1.14). $\mathrm{PM}_{2.5}$ from soil/road dust was found to be associated with hyperglycemia (RR: 1.12; 95\% CI: 1.06-1.19) and hypertension (RR: 1.11; 95\% CI: 1.05-1.18), while $\mathrm{PM}_{2.5}$ from traffic was associated with hyperglycemia (RR: $1.33 ; 95 \%$ CI: 1.05-1.71). We did not observe any health associations with source-specific mass exposures. Our findings suggest that exposure to specific elemental components of $\mathrm{PM}_{2.5}$, especially $\mathrm{Ni}$, may contribute to the development of cardiometabolic disorders.
\end{abstract}

Keywords: air pollution; particulate matter; metabolic syndrome; hypertension; diabetes; hyperglycemia

\section{Introduction}

Jeddah, a major metropolitan city of nearly 3.4 million residents, serves as a commercial, economic and cultural hub of Saudi Arabia and the Middle East [1]. Rapid population growth and expansion of the city have resulted in deteriorating air quality, raising concerns about the potential health effects.

Ambient air pollution is linked with adverse health effects, with numerous studies establishing causal associations between exposure to particulate matter (PM) and elevated risk for cardiovascular morbidity and mortality [2]. Preliminary epidemiological and mechanistic evidence have suggested that ambient air pollution exposure is also involved in the development of cardiometabolic disorders [3]. Metabolic syndrome (MetS) is a cluster of risk factors, with the Adult Treatment Panel III formally defining MetS as having at least three of the five out of hypertension, abdominal obesity, elevated 
fasting glucose, high serum triglycerides and low circulating high-density lipoprotein [4]. MetS is a precursor of developing cardiovascular disease and type II diabetes mellitus, and the presence of component abnormalities of MetS may impart individuals with greater susceptibility to PM-related health effects [5]. The prevalence of MetS has rapidly risen in Saudi Arabia over the past two decades, with studies estimating prevalence in the country as high as $41 \%$ [6].

Past epidemiologic investigation of the association between air pollution and cardiometabolic disorders have shown mixed, but generally positive associations. For example, short [7], long [8], long and short [9], intermittent [10]. Exposure to PM with an aerodynamic diameter equal to or less than $2.5 \mu \mathrm{m}\left(\mathrm{PM}_{2.5}\right)$ was found to contribute to increased incidence and/or prevalence of diabetes mellitus (DM) among U.S. adults. Short-term exposure to $\mathrm{PM}_{2.5}$ has been found to be associated with immediate elevation of blood pressure [11,12]. Long-term exposure has been associated with the development of hypertension [13]. Associations between air pollution and formally defined MetS have not been examined extensively to date, although recently a significant association between $\mathrm{PM}_{10}$ and prevalence of MetS, defined via multiple definitions, was reported in a cohort of Swiss adults [14].

In animal studies, exposure to $\mathrm{PM}_{2.5}$ increased blood glucose, adipose inflammation, and insulin resistance [15]. It impaired energy metabolism and increased inflammation in insulin responsive organs, resulting in imbalances in circulating leptin and adiponectin levels [16]. By triggering autonomic nervous system imbalance, $\mathrm{PM}_{2.5}$ exposure also promoted vasoconstriction and reduced insulin sensitivity [17]. $\mathrm{PM}_{2.5}$ impaired renal handling of excess sodium, inhibiting normal nocturnal reduction in blood [18].

As PM represents a complex mixture that is directly released from both geogenic and anthropogenic sources, or formed in the atmosphere from pollutant gases into secondary aerosols through chemical reactions, the chemical and physical characteristics, as well as the associated health effects, of the PM air pollution mixture are variable depending on its source(s), and this is thought to also influence PM toxicity [19]. Only a few studies have partitioned the health effects to source-related PM components [20-22], and none that we are aware of have been conducted to date in the Middle East.

In this innovative study, based upon our prior quantification of elemental components and source apportionment of PM with an aerodynamic diameter equal to or less than $10 \mu \mathrm{m}$ in size $\left(\mathrm{PM}_{10}\right)$ and $\mathrm{PM}_{2.5}$ in Jeddah [23], we investigated the epidemiological relationships of the previously estimated PM exposures from various sources in Jeddah with the prevalence of MetS in a local cohort, as well as with three component cardiometabolic abnormalities (hyperglycemia, hypertension, and obesity), within this recruited cohort.

\section{Materials and Methods}

\subsection{Air Quality Measurements in Jeddah}

The primary mobile source of pollution in Jeddah is traffic, with more than 1.4 million vehicles fueled mainly by unleaded gasoline and diesel oil, while major stationary sources in Jeddah include an oil refinery, a major seaport, a desalinization plant, a power generation plant, and several manufacturing industries (Figure 1). As elaborated in more details in Khodeir et al. [23], air samplers were installed at seven locations across Jeddah. Samples were collected every $24 \mathrm{~h}$ from midnight to midnight every other day, using $\mathrm{PM}_{10}$ and $\mathrm{PM}_{2.5}$ Harvard impactors [24] and calibrated vacuum pumps to draw air at a rate of $10.0 \mathrm{~L} / \mathrm{min}$ onto Teflon filters (GelmanTeflo, $37 \mathrm{~mm}, 0.2 \mu \mathrm{m}$ pore-size). The seven sampling sites were located throughout the city (Figure 1), and were selected based on varying traffic and/or population density: four in urban areas (University Campus, Al-Nuzlah, Pitrumin, and Al-Rughama), and three in residential areas (Al-Muhammadiyah, Al-Rehab and Al-Alfiyyah). 


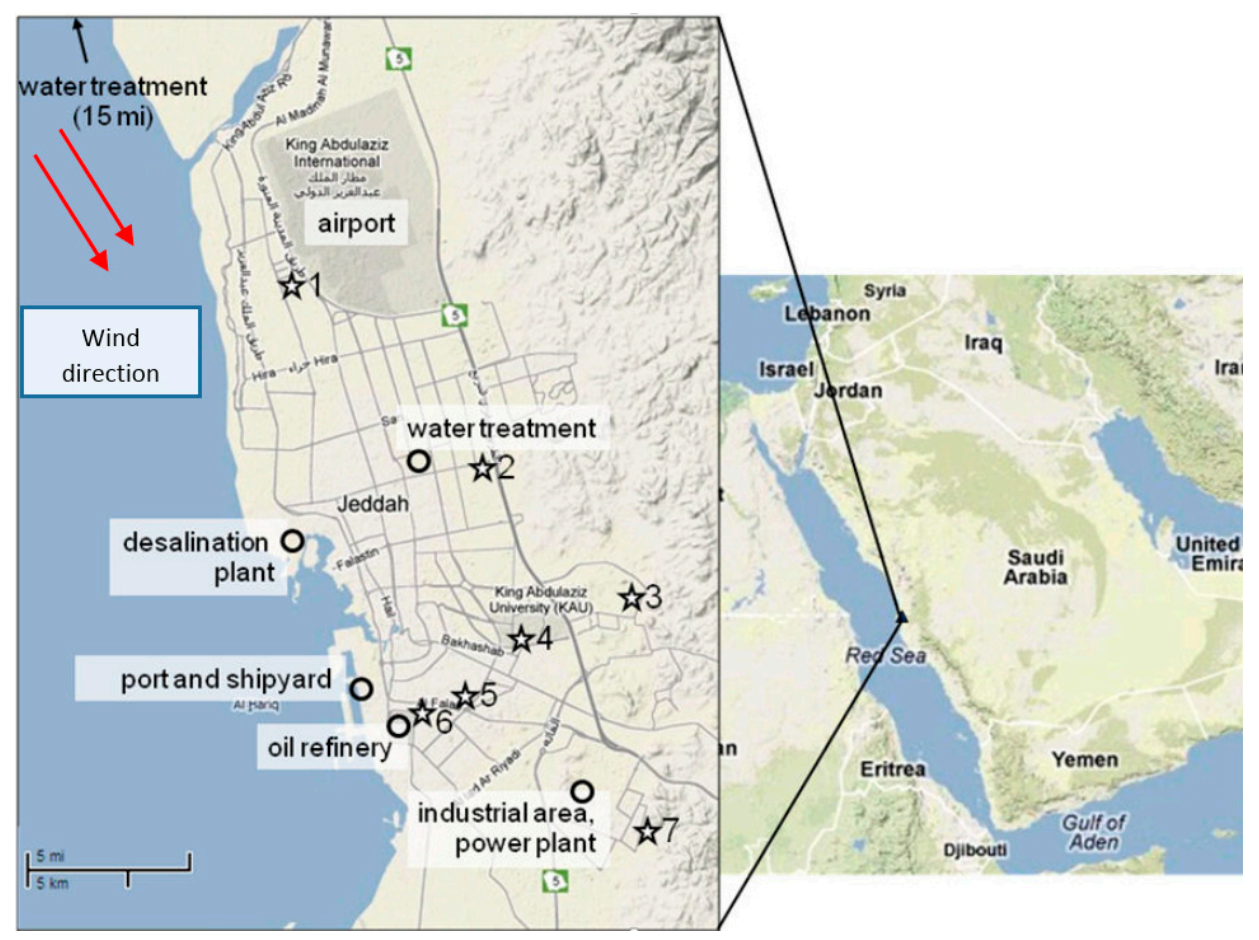

Figure 1. Locations of sampling sites (stars) and notable stationary industrial sources (circles) in Jeddah; 1 = Al-Muhammadiyah; 2 = Al-Rehab; 3 = Al-Rughama; 4 = University Campus; 5 = Al-Nuzlah Al Yamaneyyah; 6 = Pitrumin; 7 = Al-Alfiyyah.

\subsection{Source Identification}

We applied the widely employed Absolute Principal Components Analysis (APCA) method developed by Thurston and Spengler [25] in order to identify major PM source categories in each size fraction. This provided positive indices of daily source impacts, upon which daily PM mass concentrations were regressed to achieve a source apportionment for application to this research. Estimates of mass contributed by the identified source categories for each of the locations were calculated using factor weights, and the regression models developed in a previous source apportionment study conducted in Jeddah [23]. For $\mathrm{PM}_{2.5}$, four source factors were identified based on the factor eigenvalues and factor source interpretability as follows: soil/road dust (loadings on $\mathrm{Al}$, $\mathrm{Ca}, \mathrm{Cr}, \mathrm{Fe}, \mathrm{K}, \mathrm{Mg}, \mathrm{Mn}, \mathrm{Si}, \mathrm{Sr}, \mathrm{Ti})$, residual oil (Ni, S, V), solid waste incineration $(\mathrm{Cu}, \mathrm{Zn})$, and traffic $(\mathrm{Pb}$ and $\mathrm{S})$; for $\mathrm{PM}_{10}$, three factors were identified: soil/road dust, traffic, and solid waste incineration.

\subsection{Study Population Recruitment, Exposure Assessment, and Health Measurements}

Participants ( $\mathrm{N}=2686$ ) were recruited from interviewing individuals at two mega-malls, one located in North Jeddah, and the other located in South Jeddah, from June 2011 to May 2012. Participants were enrolled by trained personnel based on their willingness to participate in the study, excluding only those having lived in the area of study for less than 15 years. Confidentiality was maintained in the completed questionnaires, and the identity of the participants remained anonymous. The questionnaire included information on age, type of residence, occupation, education, smoking habits, sleeping hours, physical activity, hours since the last meal, past history of diabetes, hypertension, and other chronic diseases, and weekly frequency of food intakes by type. Physical activity was defined as 'yes' if participants were involved in walking, swimming, running, and biking. After the interview, body weight and height were measured without shoes using electronic measuring scale. Body mass index (BMI) was calculated as weight in kilograms divided by height in $\mathrm{m}^{2}$ and obesity was defined as BMI > 30 for both men and women. Blood pressure was monitored in three measurements taken 
in 5-min intervals and averaged. Hypertension was defined as systolic blood pressure $\geq 140 \mathrm{mmHg}$ and/or diastolic blood pressure $\geq 90 \mathrm{mmHg}$; those reporting use of anti-hypertensive drugs were considered as hypertensive regardless of their recorded blood pressure. Random blood sugar levels were measured and hyperglycemia was defined if the values were equal to or exceeded $126 \mathrm{mg} / \mathrm{dL}$; those reporting use of diabetes control medications were considered as having diabetes regardless of recorded blood sugar levels. Presence of MetS was defined as having all three of the cardiometabolic abnormalities measured in the study: obesity, hypertension, and hyperglycemia [26]. Individual exposure to air pollution was linked to the subjects' district of residence, using averaged values from the samplers that were installed at seven sites throughout Jeddah districts as described previously (Figure 1). An informed verbal consent was obtained from each participant, and the study proposal was approved by the King Abdulaziz University Research Ethics Committee (number 700-12).

\subsection{Statistical Analyses}

The associations between PM concentration levels and prevalence of MetS, as well as with individual components of MetS, were investigated using a generalized linear mixed effects model, adjusted for each individual's age, sex, type of residence (house, apartment, villa), marriage status (currently married/no), occupation (unemployed, labor work, desk work), education level (<secondary, secondary, university and post graduate), physical activity (no/yes), diet (weekly consumption of fresh fruits and vegetables), current smoking status (currently smoking/no), and district location (as a random effect). Detailed cohort characteristics, by location of residence, are displayed in Table 1. Subjects with incomplete individual covariates data were excluded, the total number of subjects available for analysis was $\mathrm{N}=2025$. All statistical analyses were conducted in SAS (R) Studio 3.1 (SAS Institute Inc., Cary, NC, USA). 
Table 1. Study cohort characteristics by location.

\begin{tabular}{|c|c|c|c|c|c|c|c|c|c|}
\hline & & Total & Al-Nuzlah & Al-Alfiyya & Al-Rehab & Pitrumin & Al-Rughama & University & Al-Muhammadiyah \\
\hline & & $N=2025$ & $\mathrm{~N}=\mathbf{2 6 6}$ & $\mathrm{N}=157$ & $\mathrm{~N}=409$ & $\mathrm{~N}=104$ & $N=103$ & $\mathrm{~N}=697$ & $\mathrm{~N}=\mathbf{2 9 8}$ \\
\hline Age & Mean (SD) & $31.46(10.69)$ & $29.43(11.36)$ & $35.26(11.73)$ & $32.28(10.41)$ & $30.66(10.80)$ & $28.38(8.73)$ & $31.37(10.98)$ & $31.75(9.06)$ \\
\hline \multirow{2}{*}{ Sex } & Male N (\%) & $996(49.2)$ & $185(70.1)$ & $130(86.1)$ & $175(42.8)$ & $42(40.4)$ & $61(59.2)$ & 246 (35.3) & $157(52.9)$ \\
\hline & Female N $(\%)$ & $1029(50.8)$ & $79(29.9)$ & $21(13.9)$ & $234(57.2)$ & $62(59.6)$ & $42(40.8)$ & $451(64.7)$ & $140(47.1)$ \\
\hline \multirow{2}{*}{ Smoking } & Non-smoker N (\%) & $1493(73.7)$ & $219(82.9)$ & $103(68.2)$ & $296(72.4)$ & $80(76.9)$ & $59(57.3)$ & 526 (75.5) & $210(70.7)$ \\
\hline & Smoker N (\%) & $532(26.3)$ & $45(17.0)$ & $48(31.8)$ & $113(27.6)$ & $24(23.1)$ & $44(42.7)$ & $171(24.5)$ & $87(29.3)$ \\
\hline \multirow{3}{*}{$\begin{array}{l}\text { Fruit and Vegetable } \\
\text { Intake }\end{array}$} & $\leq 4$ times/week $\mathrm{N}(\%)$ & 959 (47.4) & $164(62.1)$ & $88(58.3)$ & $143(35.0)$ & $46(44.2)$ & 49 (476.) & $342(49.1)$ & $127(42.8)$ \\
\hline & 5-6 times/week N (\%) & $787(38.9)$ & $82(31.1)$ & $55(36.4)$ & 170 (41.6) & $49(47.1)$ & $39(37.9)$ & $276(39.6)$ & $116(39.1)$ \\
\hline & $\geq 7$ times/week $\mathrm{N}(\%)$ & $279(13.8)$ & $18(6.8)$ & $9(5.3)$ & $96(23.5)$ & $9(8.6)$ & $15(14.6)$ & 79 (11.3) & $54(18.2)$ \\
\hline \multirow{3}{*}{ Residence } & House N (\%) & $681(40.0)$ & $152(57.6)$ & $25(16.6)$ & $92(22.5)$ & $74(71.2)$ & $58(56.3)$ & $221(31.7)$ & $59(19.9)$ \\
\hline & Apartment N (\%) & $1261(68.9)$ & $108(40.9)$ & $87(57.6)$ & 301 (73.6) & $30(28.8)$ & $44(42.7)$ & $467(67.0)$ & $224(75.4)$ \\
\hline & Villa N (\%) & $83(3.1)$ & $4(1.5)$ & $39(25.8)$ & $16(3.9)$ & $0(0.0)$ & $1(0.1)$ & $9(1.3)$ & $14(4.7)$ \\
\hline \multirow{2}{*}{ Marietal Status } & No N (\%) & $971(48.0)$ & $164(62.1)$ & $44(29.1)$ & $163(39.9)$ & $57(54.8)$ & $63(61.2)$ & $351(50.3)$ & 129 (43.4) \\
\hline & Yes $\mathrm{N}(\%)$ & $1054(52.0)$ & $100(37.9)$ & $107(70.9)$ & $246(60.1)$ & $47(45.2)$ & $40(38.8)$ & $346(49.6)$ & 168 (56.6) \\
\hline \multirow{3}{*}{ Type of Work } & No Work N (\%) & $523(25.8)$ & $73(27.7)$ & $30(19.9)$ & $146(35.7)$ & $32(30.8)$ & $23(22.3)$ & 129 (18.5) & $90(30.3)$ \\
\hline & Labor Work N (\%) & $1339(66.1)$ & $160(60.6)$ & $76(50.3)$ & $223(54.5)$ & $69(66.3)$ & $80(77.7)$ & $562(80.6)$ & $169(56.9)$ \\
\hline & Desk Work N $(\%)$ & $163(8.1)$ & $31(11.7)$ & $45(29.8)$ & $40(9.8)$ & $3(2.9)$ & $0(0.0)$ & $6(0.9)$ & $38(12.8)$ \\
\hline \multirow{7}{*}{ Education Level } & Illiterate $\mathrm{N}(\%)$ & $67(3.3)$ & $17(6.4)$ & $2(1.3)$ & $15(3.7)$ & $3(2.9)$ & $2(1.9)$ & $28(4.0)$ & $0(0.0)$ \\
\hline & Can Read \& Write N (\%) & $59(2.9)$ & $8(3.0)$ & $2(1.3)$ & $11(2.7)$ & $3(2.9)$ & $0(0.0)$ & $26(3.7)$ & $9(3.0)$ \\
\hline & Primary School N (\%) & $30(1.5)$ & $9(3.5)$ & $2(1.3)$ & $3(0.7)$ & $6(5.8)$ & $0(0.0)$ & $8(1.1)$ & $2(0.7)$ \\
\hline & Prepatory School N (\%) & $116(5.7)$ & $33(12.5)$ & $1(0.7)$ & $23(5.6)$ & $10(9.6)$ & $5(4.9)$ & $31(4.5)$ & $13(4.4)$ \\
\hline & Secondary School N (\%) & 758 (37.5) & $109(41.3)$ & $52(34.4)$ & $172(42.1)$ & $43(41.3)$ & $38(36.9)$ & $230(32.9)$ & $114(38.4)$ \\
\hline & University Degree N $(\%)$ & $969(47.9)$ & $85(32.2)$ & $90(59.6)$ & $174(42.5)$ & $38(36.5)$ & $57(55.3)$ & $369(52.9)$ & $156(52.5)$ \\
\hline & Post Graduate N (\%) & $26(1.3)$ & $3(1.1)$ & $2(1.3)$ & $11(2.7)$ & $1(1.0)$ & $1(1.0)$ & $5(0.7)$ & $3(1.0)$ \\
\hline \multirow{2}{*}{ Physical Activity } & No N (\%) & $1315(64.9)$ & $143(64.2)$ & $37(24.5)$ & $323(78.9)$ & $56(53.8)$ & $54(52.5)$ & 496 (71.2) & 206 (69.4) \\
\hline & Yes N (\%) & $710(35.1)$ & $121(45.8)$ & $114(75.5)$ & $86(21.0)$ & $48(46.2)$ & $49(47.5)$ & $201(28.8)$ & $91(30.6)$ \\
\hline \multirow{2}{*}{ Metabolic Syndrome } & No N (\%) & $1656(81.8)$ & $232(87.9)$ & $133(88.1)$ & $290(70.9)$ & $86(82.7)$ & $91(88.3)$ & $607(87.1)$ & 217 (73.1) \\
\hline & Yes N (\%) & $369(18.2)$ & $32(12.1)$ & $18(11.9)$ & $119(29.1)$ & $18(17.3)$ & $12(11.7)$ & $90(12.9)$ & $80(26.9)$ \\
\hline \multirow{2}{*}{ Hyperglycemia } & No N (\%) & $941(46.5)$ & $186(70.5)$ & $122(80.8)$ & $215(52.6)$ & $77(74.0)$ & $74(71.8)$ & 491 (70.4) & 158 (53.2) \\
\hline & Yes N (\%) & $1084(53.5)$ & $78(29.5)$ & $29(19.2)$ & $194(47.4)$ & $27(26.0)$ & $29(28.2)$ & $206(29.6)$ & $139(46.8)$ \\
\hline \multirow{2}{*}{ Hypertension } & No N (\%) & $1323(65.3)$ & $132(50.0)$ & $86(56.9)$ & $147(35.9)$ & $45(43.3)$ & $45(43.7)$ & 357 (51.2) & 129 (43.4) \\
\hline & Yes N (\%) & $702(34.7)$ & $132(50.0)$ & $65(43.1)$ & $262(64.1)$ & $59(56.7)$ & $58(56.3)$ & $340(48.8)$ & $168(56.6)$ \\
\hline \multirow{2}{*}{ Obesity } & No N (\%) & $659(32.5)$ & $112(42.4)$ & $64(42.4)$ & $129(31.5)$ & $29(27.9)$ & $41(39.8)$ & $226(32.4)$ & 58 (19.5) \\
\hline & Yes N (\%) & $1366(67.5)$ & $152(57.6)$ & 87 (57.6) & $280(68.5)$ & $75(72.1)$ & $62(60.2)$ & $471(67.6)$ & $239(80.5)$ \\
\hline
\end{tabular}




\section{Results and Discussion}

Summary characteristics of the seven monitoring sites are presented in Table 2. As seen in Table 2, the overall average concentrations of $\mathrm{PM}_{2.5}\left(38.6 \mu \mathrm{g} / \mathrm{m}^{3}, \mathrm{SD}=21.4 \mu \mathrm{g} / \mathrm{m}^{3}\right)$ and $\mathrm{PM}_{10}\left(85.1 \mu \mathrm{g} / \mathrm{m}^{3}\right.$, $\mathrm{SD}=30.30 \mu \mathrm{g} / \mathrm{m}^{3}$ ) measured in the present study period across the sampling sites greatly exceeded the recommended annual levels by the World Health Organization for $\mathrm{PM}_{2.5}\left(10 \mu \mathrm{g} / \mathrm{m}^{3}\right)$ and $\mathrm{PM}_{10}$ $\left(20 \mu \mathrm{g} / \mathrm{m}^{3}\right)$ [27]. Highest concentrations for $\mathrm{PM}_{2.5}\left(73.2 \mu \mathrm{g} / \mathrm{m}^{3}\right)$ and $\mathrm{PM}_{10}\left(141.3 \mu \mathrm{g} / \mathrm{m}^{3}\right)$ were observed in the Al-Rughama, while lowest concentrations for $\mathrm{PM}_{2.5}\left(15.8 \mu \mathrm{g} / \mathrm{m}^{3}\right)$ and $\mathrm{PM}_{10}\left(47.0 \mu \mathrm{g} / \mathrm{m}^{3}\right)$ were observed in Al-Muhammadiyah. Al-Rughama is a suburban area characterized by intensive traffic, construction activities, small workshops and open burning of solid waste, while al-Muhammadiyah is a quiet typical residential area with light traffic.

Sulfur $\left(3.39 \mu \mathrm{g} / \mathrm{m}^{3}\right)$ was the dominant element found in $\mathrm{PM}_{2.5}$, representing $26.8 \%$ of total elemental mass, whereas silicon $\left(10.4 \mu \mathrm{g} / \mathrm{m}^{3}\right)$ was the dominant element found in $\mathrm{PM}_{10}$, representing $34.53 \%$ of total elemental mass. The elemental components profiles of each location generally reflected the environmental and physical characteristics of their respective surroundings; samples from locations closest to the non-developed lands east of the city-Al-Alfiyyah, Al-Rehab, and Al-Rughama-had elevated concentrations of crustal elements, such as $\mathrm{Al}, \mathrm{Ca}, \mathrm{Mg}$, and $\mathrm{Si}$. High concentrations of $\mathrm{Ni}, \mathrm{S}$, and V, markers of residual oil combustion were measured in Pitrumin, Al-Nuzlah and Al-Rughama, which are the sampling locations closest to the port and oil refinery. Al-Rehab and Al-Alfiyyah, closest to water treatment and industrial power plant areas, had high concentrations of $\mathrm{Cu}$ and $\mathrm{Zn}$, elements commonly associated with open and/or industrial waste burning. Samples from Al-Rughama, Al-Alfiyahh, and University, which are located nearby the major highway, had elevated concentrations of $\mathrm{Pb}$, a marker for traffic even after discontinued leaded gasoline use, due to accumulation in soil [28].

The degree of metal contamination in atmospheric $\mathrm{PM}_{2.5}$ and $\mathrm{PM}_{10}$ of the study areas can be assessed by comparing their measured concentrations with regulatory standards. Such an approach, however, cannot be adopted in the present study because standards are not available for the measured metals. An alternative approach would be to compare the heavy metal concentrations in the study area with the safe limits proposed by international agencies. World Health Organization (WHO) [27] standards for atmospheric $\mathrm{Pb}, \mathrm{Mn}, \mathrm{Cr}$ and $\mathrm{Ni}$ are 500, 150, 1100 and $0.38 \mathrm{ng} / \mathrm{m}^{3}$, while those reported by the Agency for Toxic Substances and Disease Registry (ATSDR) [29] are 1500, 500, 100 and 0.24 ng/m $\mathrm{m}^{3}$ for the same metals in the same order. In the present study, only Ni levels in both $\mathrm{PM}_{2.5}$ and $\mathrm{PM}_{10}$ were many times higher than the proposed WHO and ATSDR standards, and $\mathrm{Mn}$ in $\mathrm{PM}_{10}$ at Al-Rughama was higher than $\mathrm{WHO}$ standards. The average concentrations of $\mathrm{Pb}, \mathrm{Cr}$ and $\mathrm{Mn}$ were even lower than the WHO and ATSDR standards. Associations between ambient air nickel and cardiovascular disease have become a research issue in the new millennium [21,30-32]. At the molecular level and for Saudi Arabia specifically, Brocato et al. [33] reported that in vivo exposures to particulate matter collected from Jeddah or nickel chloride display similar dysregulation of metabolic syndrome genes.

Results from the statistical modeling are presented in Table 3. After adjusting for confounders, we found that $\mathrm{PM}_{10}$ was not associated with MetS (RR: 1.03; 95\% CI: 0.95-1.13), hyperglycemia (RR: 0.98; 95\% CI: 0.92-1.05), hypertension (RR: 1.06; 95\% CI: 1.00-1.13), and BMI (RR: 0.95; 95\% CI: 0.89-1.02). This is not in accordance with the results of Brocato et al. [34] who reported that acutely exposing mice to $\mathrm{PM}_{10}$ collected from Jeddah induced genes involved in inflammation, cholesterol and lipid metabolism, and atherosclerosis. On the other hand, we found that a $10 \mu \mathrm{g} / \mathrm{m}^{3}$ increase in $\mathrm{PM}_{2.5}$ was significantly associated with MetS prevalence (RR: 1.12; 95\% CI: 1.06-1.19), hyperglycemia (RR: 1.08; 95\% CI: 1.03-1.13) and hypertension (RR: 1.09; 95\% CI: 1.01-1.18), but not with BMI (RR: 0.95; 95\% CI: 0.91-1.00). This proves that, for chronic exposure, $\mathrm{PM}_{10}$ is less risky concerning cardio metabolic effects. 
Table 2. PM and elemental constituents concentration levels (Mean \pm S.D., $\mu \mathrm{g} / \mathrm{m}^{3}$ ) by location.

\begin{tabular}{|c|c|c|c|c|c|c|c|c|}
\hline & \multicolumn{2}{|c|}{ Total } & \multicolumn{2}{|c|}{ Al-Nuzlah } & \multicolumn{2}{|c|}{ Al-Alfiyya } & \multicolumn{2}{|c|}{ Al-Rehab } \\
\hline & $\mathbf{P M}_{2.5}$ & $\mathrm{PM}_{10}$ & $\mathbf{P M}_{2.5}$ & $\mathrm{PM}_{10}$ & $\mathbf{P M}_{2.5}$ & $\mathrm{PM}_{10}$ & $\mathbf{P M}_{2.5}$ & $\mathbf{P M}_{10}$ \\
\hline PM & 29.07 (18.68) & $85.12(30.30)$ & $29.10(14.11)$ & 73.51 (10.13) & 24.51 (11.77) & $99.42(43.94)$ & $18.04(3.96)$ & 68.95 (20.48) \\
\hline $\mathrm{Al}$ & $0.89(1.35)$ & $3.42(2.05)$ & $0.28(0.066)$ & $2.06(0.46)$ & $0.58(0.36)$ & $4.79(2.50)$ & $0.28(0.12)$ & $2.41(0.86)$ \\
\hline $\mathrm{Ca}$ & $0.63(0.77)$ & 4.18 (1.33) & $0.31(0.037)$ & $3.55(0.49)$ & $0.36(0.18)$ & $4.48(2.35)$ & $0.28(0.18)$ & $3.48(1.32)$ \\
\hline $\mathrm{Cr}$ & $0.0028(0.0031)$ & $0.0093(0.0058)$ & $0.0018(0.00077)$ & $0.0066(0.0011)$ & $0.0021(0.00087)$ & $0.013(0.0073)$ & $0.0014(0.00099)$ & $0.0059(0.0023)$ \\
\hline $\mathrm{Cu}$ & $0.0061(0.0041)$ & $0.017(0.0076)$ & $0.0024(0.0023)$ & $0.012(0.0048)$ & $0.013(0.0097)$ & $0.022(0.015)$ & $0.0018(0.0014)$ & $0.013(0.0038)$ \\
\hline $\mathrm{Fe}$ & $0.72(1.18)$ & $3.32(2.24)$ & $0.17(0.027)$ & $1.84(0.38)$ & $0.37(0.21)$ & $4.25(2.43)$ & $0.23(0.15)$ & $2.29(0.86)$ \\
\hline K & $0.21(0.17)$ & $0.72(0.27)$ & $0.15(0.033)$ & $0.52(0.083)$ & $0.013(0.063)$ & $0.86(0.51)$ & $0.11(0.030)$ & $0.56(0.19)$ \\
\hline $\mathrm{Mg}$ & $0.33(0.43)$ & $1.44(0.52)$ & $0.20(0.079)$ & $1.17(0.16)$ & $0.23(0.15)$ & $1.93(0.87)$ & $0.11(0.057)$ & $1.21(0.54)$ \\
\hline $\mathrm{Mn}$ & $0.022(0.033)$ & $0.10(0.063)$ & $0.0054(0.0018)$ & $0.053(0.012)$ & $0.012(0.0057)$ & $0.12(0.062)$ & 0.009 (0.0048) & $0.069(0.028)$ \\
\hline $\mathrm{Ni}$ & $0.0071(0.0028)$ & 0.011 (0.0039) & $0.0071(0.0023)$ & 0.099 (0.0027) & $0.0064(0.0033)$ & $0.012(0.0067)$ & $0.0042(0.00091)$ & $0.0073(0.0025)$ \\
\hline $\mathrm{Pb}$ & $0.13(0.14)$ & $0.16(0.17)$ & $0.037(0.049)$ & $0.039(0.044)$ & $0.49(0.44)$ & $0.47(0.47)$ & $0.086(0.14)$ & $0.11(0.15)$ \\
\hline $\mathrm{S}$ & $3.48(0.75)$ & $3.39(0.51)$ & $4.04(0.45)$ & $3.76(0.47)$ & $3.87(2.34)$ & $3.47(2.17)$ & $2.51(0.35)$ & $2.69(0.15)$ \\
\hline $\mathrm{Si}$ & 2.47 (3.99) & $11.19(6.70)$ & $0.72(0.23)$ & $7.08(1.36)$ & $1.44(0.89)$ & $15.29(8.10)$ & $0.68(0.43)$ & $7.69(2.80)$ \\
\hline $\mathrm{Sr}$ & $0.0049(0.0061)$ & $0.026(0.0085)$ & $0.0021(0.00066)$ & $0.022(0.0034)$ & $0.003(0.0019)$ & $0.029(0.015)$ & $0.0018(0.0020)$ & $0.023(0.015)$ \\
\hline $\mathrm{Ti}$ & $0.070(0.13)$ & $0.32(0.25)$ & $0.011(0.0027)$ & $0.15(0.028)$ & $0.032(0.021)$ & $0.42(0.26)$ & $0.017(0.014)$ & $0.21(0.08)$ \\
\hline $\mathrm{V}$ & $0.025(0.010)$ & $0.032(0.012)$ & $0.029(0.0098)$ & $0.33(0.0096)$ & $0.023(0.012)$ & $0.031(0.015)$ & $0.015(0.0038)$ & $0.021(0.06)$ \\
\hline \multirow[t]{3}{*}{$\mathrm{Zn}$} & $0.038(0.023)$ & $0.073(0.044)$ & $0.017(0.0069)$ & $0.039(0.012)$ & $0.056(0.064)$ & $0.13(0.17)$ & $0.019(0.0088)$ & $0.049(0.011)$ \\
\hline & \multicolumn{2}{|c|}{ Pitrumin } & \multicolumn{2}{|c|}{ Al-Rughama } & \multicolumn{2}{|c|}{ University } & \multicolumn{2}{|c|}{ Al-Muhammadiyah } \\
\hline & $\mathbf{P M}_{2.5}$ & $\mathrm{PM}_{10}$ & $\mathbf{P M}_{2.5}$ & $\mathrm{PM}_{10}$ & $\mathbf{P M}_{2.5}$ & $\mathrm{PM}_{10}$ & $\mathbf{P M}_{2.5}$ & $\mathbf{P M}_{10}$ \\
\hline PM & $31.14(5.85)$ & 107.13 (28.75) & 73.16 (65.08) & $141.27(124.20)$ & 29.91 (11.67) & 85.67 (33.09) & $15.78(3.05)$ & $47.01(6.52)$ \\
\hline $\mathrm{Al}$ & $0.41(0.22)$ & $3.51(1.07)$ & $3.93(4.07)$ & 7.17 (6.07) & $0.63(1.02)$ & 3.07 (1.77) & $0.15(0.43)$ & $0.91(0.41)$ \\
\hline $\mathrm{Ca}$ & $0.53(0.17)$ & $6.11(1.42)$ & $2.36(2.76)$ & $4.95(2.94)$ & $0.39(0.30)$ & $4.77(2.18)$ & $0.19(0.15)$ & $1.96(0.61)$ \\
\hline $\mathrm{Cr}$ & $0.0015(0.0010)$ & $0.0089(0.0031)$ & $0.0097(0.010)$ & $0.021(0.020)$ & $0.0019(0.0021)$ & $0.0083(0.0053)$ & $0.00092(0.00058)$ & $0.0022(0.00081)$ \\
\hline $\mathrm{Cu}$ & $0.0054(0.0053)$ & $0.029(0.0096)$ & $0.0098(0.0057)$ & $0.016(0.011)$ & $0.0077(0.011)$ & $0.019(0.016)$ & $0.0029(0.0037)$ & $0.0048(0.0022)$ \\
\hline $\mathrm{Fe}$ & $0.36(0.15)$ & $3.59(1.16)$ & $3.37(3.94)$ & $7.71(7.73)$ & $0.39(0.64)$ & $2.81(1.59)$ & $0.12(0.093)$ & $0.75(0.22)$ \\
\hline $\mathrm{K}$ & $0.21(0.091)$ & $0.91(0.29)$ & $0.59(0.62)$ & $1.14(0.93)$ & $0.16(0.13)$ & $0.69(0.31)$ & $0.12(0.024)$ & $0.35(0.075)$ \\
\hline $\mathrm{Mg}$ & $0.19(0.098)$ & $1.55(0.47)$ & $1.29(1.21)$ & $2.21(1.51)$ & $0.25(0.33)$ & $1.41(0.60)$ & $0.058(0.025)$ & $1.55(0.16)$ \\
\hline $\mathrm{Mn}$ & 0.014 (0.0073) & $0.11(0.035)$ & $0.097(0.11)$ & $0.23(0.21)$ & $0.015(0.021)$ & $0.095(0.052)$ & $0.0051(0.0028)$ & $0.11(0.0079)$ \\
\hline $\mathrm{Ni}$ & $0.012(0.0048)$ & $0.018(0.0044)$ & $0.0097(0.0058)$ & $0.13(0.0088)$ & $0.0058(0.0022)$ & $0.011(0.0044)$ & $0.0043(0.00096)$ & $0.018(0.0021)$ \\
\hline $\mathrm{Pb}$ & $0.031(0.020)$ & $0.041(0.022)$ & $0.17(0.27)$ & $0.021(0.34)$ & $0.21(0.42)$ & $0.27(0.52)$ & $0.0066(0.0067)$ & $0.0093(0.0063)$ \\
\hline $\mathrm{S}$ & $4.51(0.97)$ & $4.06(0.67)$ & $3.65(1.32)$ & $2.82(1.25)$ & $3.11(1.19)$ & $3.71(0.99)$ & $2.63(0.47)$ & $3.19(0.73)$ \\
\hline $\mathrm{Si}$ & $1.16(0.62)$ & $12.18(3.96)$ & 11.49 (12.57) & $23.35(20.07)$ & $1.47(2.46)$ & 10.07 (5.42) & $0.34(0.21)$ & $2.64(1.04)$ \\
\hline $\mathrm{Sr}$ & $0.0044(0.0017)$ & $0.036(0.021)$ & $0.019(0.022)$ & $0.036(0.025)$ & $0.0029(0.0027)$ & $0.027(0.014)$ & 0.0021 (0.00097) & $0.012(0.003)$ \\
\hline $\mathrm{Ti}$ & $0.025(0.014)$ & $0.31(0.095)$ & $0.36(0.43)$ & $0.83(0.90)$ & $0.034(0.069)$ & $0.25(0.17)$ & $0.012(0.0094)$ & $0.07(0.03)$ \\
\hline $\mathrm{V}$ & $0.045(0.0099)$ & $0.055(0.0072)$ & $0.028(0.015)$ & $0.031(0.014)$ & $0.019(0.0069)$ & 0.029 (0.0099) & $0.016(0.0037)$ & $0.021(0.0078)$ \\
\hline $\mathrm{Zn}$ & $0.073(0.13)$ & $0.13(0.17)$ & $0.043(0.027)$ & $0.061(0.036)$ & $0.044(0.0 .76)$ & $0.079(0.13)$ & $0.0099(0.0044)$ & $0.022(0.005)$ \\
\hline
\end{tabular}


Table 3. Risk Ratios (RR) and 95\% Confidence Interval (CI) per $10 \mu \mathrm{g} / \mathrm{m}^{3}$ increment of particulate matter (PM) and factor mass estimates.

\begin{tabular}{lcccc}
\hline & Metabolic Syndrome & Hyperglycemia & Hypertension & BMI \\
\hline $\mathrm{PM}_{2.5}$ & $1.11(1.05-1.18)$ & $1.08(1.03-1.14)$ & $1.09(1.04-1.14)$ & $0.95(0.91-1.00)$ \\
Factor 1: Soil \& Road Dust & $1.16(1.08-1.25)$ & $1.12(1.06-1.19)$ & $1.11(1.05-1.18)$ & $0.95(0.90-1.01)$ \\
Factor 2: Residual Oil & $0.94(0.88-1.00)$ & $0.88(0.77-1.01)$ & $0.98(0.96-1.01)$ & $1.00(0.98-1.03)$ \\
Factor 3: Incineration & $0.99(0.92-1.07)$ & $0.98(0.91-1.05)$ & $1.00(0.96-1.04)$ & $0.85(0.76-0.95)$ \\
Factor 4: Traffic & $1.28(0.94-1.76)$ & $1.33(1.05-1.71)$ & $1.12(0.89-1.12)$ & $0.74(0.55-1.00)$ \\
\hline $\mathrm{PM}_{10}$ & $1.03(0.95-1.13)$ & $0.98(0.92-1.05)$ & $1.06(1.00-1.13)$ & $0.95(0.89-1.02)$ \\
Factor 1: Soil \& Road Dust & $1.03(0.95-1.10)$ & $0.99(0.95-1.05)$ & $1.05(1.00-1.10)$ & $0.99(0.89-1.10)$ \\
Factor 2: Incineration & $1.02(0.82-1.26)$ & $0.90(0.76-1.06)$ & $1.08(0.93-1.26)$ & $0.84(0.61-1.17)$ \\
Factor 3: Traffic & $1.01(0.56-1.83)$ & $1.02(0.67-1.57)$ & $0.77(0.53-1.12)$ & $1.13(0.90-1.40)$ \\
\hline
\end{tabular}

When the source of PM was studied again, none of the identified source mass estimates showed significance with neither MetS nor the specific endpoints for $\mathrm{PM}_{10}$. For $\mathrm{PM}_{2.5}$, soil/road dust mass was significantly associated with MetS (RR: 1.16; 95\% CI: 1.08-1.25), hyperglycemia (RR: 1.12; 95\% CI: 1.06-1.19), and hypertension (RR: $1.11 ; 95 \%$ CI: 1.05-1.18). This suggests that crustal elements as $\mathrm{Al}$ and $\mathrm{Si}$ originating from the surrounding deserts and partially generated as road dust may potentially be responsible for the cardiometabolic effects. Other investigations found that $\mathrm{Al}$ and Si induced markers of oxidative stress and inflammation in animals and humans [35-37], a major mechanism for the cardiovascular effects induced by particulate matter exposure [38,39]. Moreover, the deleterious role of $\mathrm{Si}$ in the development of chronic renal disease [40] might suggest its implication in hypertension. Also, a significant association (RR: 1.33; 95\% CI: 1.05-1.71) between $\mathrm{PM}_{2.5}$ traffic source components $(\mathrm{Pb}, \mathrm{S})$ and hyperglycemia was observed. Although $\mathrm{Pb}$ exposure has been falling, it is likely that even very low levels of exposure are hazardous to health [41]. Pb exposure exerts its effect on cardiovascular diseases by increasing the risk of hypertension, and through its proinflammatory properties affecting all stages of atherogenesis [42]. The actual contribution of $\mathrm{Pb}$ exposure to diabetes risk in the general population is difficult to assess. However, numerous studies proved a strong association between low levels of lead exposure and markers of oxidative stress. Oxidative stress is known to promote the development of diabetes through multiple mechanisms. Inhibition of several key components of insulin signaling pathway resulting in insulin resistance, is one of them [43]. Our source-specific findings could thus potentially explain the inconsistent results from the aforementioned studies examining the relationships between DM/hypertension incidence and an index of overall air pollution (e.g., $\mathrm{PM}_{2.5}$ mass), in which some studies observed stronger associations with $\mathrm{NO}_{2}$, a proxy for traffic-related air pollution, than $\mathrm{PM}_{2.5}$ [44,45]. While a direct effect of $\mathrm{NO}_{2}$ cannot be ruled out, results from our study suggest that elemental components generated from traffic-related $\mathrm{PM}_{2.5}$ could potentially be responsible for the observed health effects, confirming that PM might be the major component of air pollution producing the most deleterious effects on human health [46].

The major strength of the present study is that, up to our knowledge, is the first epidemiological study investigating the relationship between source-specific elemental components of PM and metabolic syndrome. However, there are several limitations of this study. First, the retrospective and cross-sectional nature of the study design precludes establishing a definitive causal link between $\mathrm{PM}_{2.5}$ exposure and the outcomes considered here. Second, several elements, carbonaceous species (elemental and organic carbon), and gases that were either not measured, or not considered in our source apportionment, could potentially be causal predictors of health outcomes of interest. For example, due to missing data in the original APCA, some elements ( $\mathrm{As}, \mathrm{Cd}, \mathrm{Cl}, \mathrm{Co}, \mathrm{Ga}$, Se, $\mathrm{Rb}$ ) were excluded in the subsequent analysis, thereby potentially influencing factor mass estimates and loadings, and in turn, the associations between source-specific mass estimates and outcomes. A lack of monitoring data for gases and/or carbonaceous species also raise the possibility that the source categories identified in our study are serving as surrogates for other unmeasured correlated constituents actually responsible for the observed adverse health effects. 


\section{Conclusions}

This is the first study to have partitioned cardiometabolic health effects of PM pollution to specific sources of PM in a Middle Eastern city, suggesting that the toxicity of PM did vary by composition. $\mathrm{PM}_{2.5}$ exposure increased the prevalence of MetS, hyperglycemia and hypertension. $\mathrm{PM}_{2.5}$ trace elements from soil/road dust, such as $\mathrm{Al}$ and $\mathrm{Si}$, were significantly associated with hyperglycemia and hypertension. Also, a significant association between $\mathrm{PM}_{2.5}$ traffic source components $(\mathrm{Pb}, \mathrm{S})$ and hyperglycemia was observed. Ni content in PM might be responsible for the observed effects. This may help decision makers to take strict measures towards limiting exposure to specified components like Ni for example. A larger and more robust study with greater spatial variability for pollutants and detailed adjustments for ecological confounding could enable a more definitive evaluation of the PM-MetS relationship.

Acknowledgments: This study was funded by King Abdulaziz University (KAU), Jeddah, under grant 4/00/00/252 and by NIEHS grant under P30ES000260. The sponsors did not have any role in the study design.

Author Contributions: Magdy Shamy, Mansour Alghamdi, Mamdouh I. Khoder, Jason Brocato, George D. Thurston, Abdularahman K. Alkhalaf, Abdullah M. Mohorjy and Max Costa designed the study and supervised execution of the work. Alser A. Alkhatim, Lung Chi Chen, Chris C. Lim executed the work and analyzed the data. Magdy Shamy, Mamdouh I. Khoder, Mansour Alghamdi and Chris C. Lim wrote the manuscript. Magdy Shamy and Mamdouh I. Khoder contributed to the manuscript preparation and proofreading. All authors approved the final version of the manuscript.

Conflicts of Interest: The authors declare no conflict of interest.

\section{References}

1. Alghamdi, M.; Alam, M.; Yin, J.; Stark, C.; Jang, E.; Harrison, R.; Shamy, M.; Khoder, K.; Shabbaj, I. Receptor modeling study of polycyclic aromatic hydrocarbons in Jeddah, Saudi Arabia. Sci. Total Environ. 2015, 506-507, 401-408. [CrossRef]

2. Brook, R.; Rajagopalan, S.; Pope, C.; Brook, J.; Bhatnagar, A.; Diez-Roux, A.; Holguin, F.; Hong, Y.; Luepker, R.; Mittleman, M.; et al. Particulate matter air pollution and cardiovascular disease: An update to the scientific statement from the American Heart Association. Circulation 2010, 121, 2331-2378. [CrossRef] [PubMed]

3. Park, S.; Auchincloss, A.; O'neill, M.; Prineas, R.; Correa, J.; Keeler, J.; Roux, A. Particulate air pollution, metabolic syndrome, and heart rate variability: The Multi-Ethnic Study of Atherosclerosis (MESA). Environ. Health Perspect. 2010, 118, 1406-1411. [CrossRef]

4. National Cholesterol Education Program (NCEP). Executive Summary of the Third Report of the National Cholesterol Education Program. Expert panel on detection, evaluation, and treatment of high blood cholesterol in adults (Adult Treatment Panel III). JAMA 2001, 285, 2486-2497.

5. Pope, C.; Turner, M.; Burnett, R.; Jerrett, M.; Gapstur, S.; Diver, W.; Krewski, D.; Brook, R. Relationships between fine particulate air pollution, cardiometabolic disorders, and cardiovascular mortality. Circ. Res. 2015, 116, 108-115. [CrossRef]

6. Alzahrani, A.; Karawagh, A.; Alshahrani, F.; Naser, T.; Ahmed, A.; Alsharef, E. Prevalence and predictors of metabolic syndrome among healthy Saudi Adults. Br. J. Diabetes Vasc. Dis. 2012, 12, 78-80. [CrossRef]

7. Pearson, J.; Bachireddy, C.; Shyamprasad, S.; Goldfine, A.; Brownstein, J. Association between fine particulate matter and diabetes prevalence in the U.S. Diabetes Care 2010, 33, 2196-2201. [CrossRef]

8. Chen, H.; Burnett, R.; Kwong, J.; Villeneuve, P.; Goldberg, M.; Brook, R.; Van Donkelaat, A.; Jerrett, M.; Martin, R.; Brook, J.; et al. Risk of incident diabetes in relation to long-term exposure to fine particulate matter in Ontario, Canada. Environ. Health Perspect. 2013, 121, 804-810. [CrossRef]

9. Park, S.; Wang, W. Ambient air pollution and type 2 diabetes mellitus: A systematic review of epidemiologic research. Curr. Environ. Health Rep. 2014, 1, 275-286. [CrossRef] [PubMed]

10. Yitshak Sade, M.; Kloog, I.; Liberty, I.; Schwartz, J.; Novack, V. The association between air pollution exposure and glucose and lipids levels. J. Clin. Endocrinol. Metab. 2016, 101, 2460-2467. [CrossRef] [PubMed] 
11. Auchincloss, A.; Diez Roux, A.; Dvonch, J.; Brown, P.; Barr, R.G.; Daviglus, M.; Goff, D.; Kaufman, G.; O'neill, M. Associations between recent exposure to ambient fine particulate matter and blood pressure in the multi-ethnic study of atherosclerosis (MESA). Environ. Health Perspect. 2008, 116, 486-491. [CrossRef] [PubMed]

12. Wu, S.; Deng, F.; Huang, J.; Wang, H.; Shima, M.; Wang, X.; Qin, Y.; Zheng, C.; Wei, H.; Hao, Y.; et al. Blood pressure changes and chemical constituents of particulate air pollution: Results from the Healthy Volunteer Natural Relocation (HVNR) Study. Environ. Health Perspect. 2013, 121, 66-72. [CrossRef] [PubMed]

13. Chen, H.; Burnett, R.; Kwong, J.; Villeneuve, P.; Goldberg, M.; Brook, R.; Van Donkelaar, A.; Jerrett, M.; Martin, R.; Kopp, A.; et al. Spatial association between ambient fine particulate matter and incident hypertension. Circulation 2014, 129, 562-569. [CrossRef] [PubMed]

14. Eze, I.; Schaffner, E.; Foraster, M.; Imboden, M.; Von Eckardstein, A.; Gerbase, M.; Rothe, T.; Rochat, T.; Kunzli, N.; Schindler, C.; et al. Long-term exposure to ambient air pollution and metabolic syndrome in adults. PLoS ONE 2015, 10, e0130337. [CrossRef] [PubMed]

15. Sun, Q.; Yue, P.; Deiuliis, J.; Lumeng, C.; Kampfrath, T.; Mikolaj, M.; Caj, Y.; Ostrowsky, M.; Parthasarathy, S.; Brook, R.; et al. Ambient air pollution exaggerates adipose inflammation and insulin resistance in a mouse model of diet-induced obesity. Circulation 2009, 119, 538-546. [CrossRef] [PubMed]

16. Liu, C.; Bai, Y.; Xu, X.; Sun, L.; Wang, A.; Wang, T.; Maurya, S.; Periasamy, M.; Morishita, M.; Harkema, J.; et al. Exaggerated effects of particulate matter air pollution in genetic type II diabetes mellitus. Part. Fibre Toxicol. 2014, 11, 27. [CrossRef] [PubMed]

17. Lindmark, S.; Wiklund, U.; Bjerle, P.; Eriksson, J. Does the autonomic nervous system play a role in the development of insulin resistance? A study on heart rate variability in first-degree relatives of type 2 diabetes patients and control subjects. Diabet. Med. 2003, 20, 399-405. [CrossRef] [PubMed]

18. Tsai, D.; Riediker, M.; Wuerzner, G.; Maillard, M.; Marques-Vidal, P.; Paccaud, F.; Vollenweider, P.; Burnier, M.; Bochud, M. Short-term increase in particulate matter blunts nocturnal blood pressure dipping and daytime urinary sodium excretion. Hypertension 2012, 60, 1061-1069. [CrossRef] [PubMed]

19. National Research Council (NRC). Research Priorities for Airborne Particulate Matter: Continuing Research Progress; National Academies Press: Washington, DC, USA, 2004.

20. Ostro, B.; Roth, L.; Malig, B.; Marty, M. The effects of fine particle components on respiratory hospital admissions in children. Environ. Health Perspect. 2009, 117, 475-480. [CrossRef] [PubMed]

21. Bell, M.; Ebisu, K.; Leaderer, B.; Gent, J.; Lee, H.; Koutrakis, P.; Wang, Y.; Dominici, F.; Peng, R. Associations between $\mathrm{PM}_{2.5}$ constituents and sources with hospital admissions: Analysis of four counties in Connecticut and Massachusetts (USA) for persons > 65 years of age. Environ. Health Perspect. 2014, 122, 138-144. [PubMed]

22. Thurston, G.; Burnett, R.; Turner, M.; Shi, Y.; Krewski, D.; Lall, R.; Ito, K.; Jerrett, M.; Gapstur, S.; Diver, W.; et al. Ischemic heart disease mortality and long-term exposure to source-related components of U.S. fine particle air pollution. Environ. Health Perspect. 2016, 124, 785-794. [CrossRef] [PubMed]

23. Khodeir, M.; Shamy, M.; Alghamdi, M.; Zhong, M.; Sun, H.; Costa, M.; Chen, L.; Maciejczyk, P. Source apportionment and elemental composition of $\mathrm{PM}_{2.5}$ and $\mathrm{PM}_{10}$ in Jeddah City, Saudi Arabia. Atmos. Pollut. Res. 2012, 3, 331-340. [CrossRef] [PubMed]

24. Turner, W.; Olson, B.; Allen, G. Calibration of sharp cut impactors for indoor and outdoor particle sampling. J. Air Waste Manag. Assoc. 2000, 50, 484-487. [CrossRef] [PubMed]

25. Thurston, G.; Spengler, J. A quantitative assessment of source contributions to inhalable particulate matter in metropolitan Boston, Massachusetts. Atmos. Environ. 1985, 19, 9-21. [CrossRef]

26. Bahijri, S.; Al Raddadi, R.; Jambi, H.; Alaamer, M.; Ferns, G. The prevalence of metabolic syndrome in an apparently healthy, normotensive and non-diabetic population in Saudi Arabia by two definitions: Implications for local practice. Open J. Endocr. Metab. Dis. 2013, 3, 18-24. [CrossRef]

27. WHO. World Health Organization. Air Quality Guidelines for Particulate Matter, Ozone, Nitrogen Dioxide and Sulfur Dioxide. Global Update. Summary of Risk Assessment 2006. Available online: http:/ /apps.who. int/iris/bitstream/10665/69477/1/WHO_SDE_PHE_OEH_06.02_eng.pdf (accessed on 13 July 2016).

28. Mielke, H.; Laidlaw, M.; Gonzales, C. Lead (Pb) legacy from vehicle traffic in eight California urbanized areas: Continuing influence of lead dust on children's health. Sci. Total Environ. 2010, 408, 3965-3975. [CrossRef] [PubMed] 
29. ATSDR. Agency for Toxic Substances and Disease Registry. Regulations and Guidelines Applicable. Available online: http:/ / www.atsdr.cdc.gov/toxprofiles/tp11-c8.pdf (accessed on 13 July 2016).

30. Peng, R.; Dominici, F.; Pastor-Barriuso, R.; Zeger, S.; Samet, S. Seasonal analyses of air pollution and mortality in 100 US cities. Am. J. Epidemiol. 2005, 161, 585-594. [CrossRef] [PubMed]

31. Dominici, F.; Peng, R.; Zeger, S.; White, R.; Samet, J. Particulate air pollution and mortality in the United States: Did the risks change from 1987 to 2000? Am. J. Epidemiol. 2007, 166, 880-888. [CrossRef] [PubMed]

32. Pun, V.; Yu, I.; Qiu, H.; Ho, K.; Sun, Z.; Louie, P.; Wong, T.; Tian, L. Short-term associations of cause-specific emergency hospitalizations and particulate matter chemical components in Hong Kong. Am. J. Epidemiol. 2014, 179, 1086-1095. [CrossRef] [PubMed]

33. Brocato, J.; Hernandez, M.; Laulicht, F.; Sun, H.; Shamy, M.; Alghamdi, M.; Khoder, M.; Kluz, T.; Chen, L.; Costa, M. In vivo exposures to particulate matter collected from Saudi Arabia or nickel chloride display similar dysregulation of metabolic syndrome genes. J. Toxicol. Environ. Health A 2015, 78, 1421-1436. [CrossRef] [PubMed]

34. Brocato, J.; Sun, H.; Shamy, M.; Kluz, T.; Alghamdi, M.; Khoder, M.; Chen, L.; Costa, M. Particulate matter from Saudi Arabia induces genes involved in inflammation, metabolic syndrome and atherosclerosis. J. Toxicol. Environ. Health A 2014, 77, 751-766. [CrossRef] [PubMed]

35. Zhang, Z.; Rong, Y.; Cui, X.; Shen, Y.; Zhou, Y.; Xiao, L.; Chen, W. Oxidative stress and mitochondrion-related cell apoptosis in human bronchial epithelial 16HBE cells induced by silica dust. Zhonghua Lao Dong Wei Sheng Zhi Ye Bing Za Zhi 2015, 33, 801-805. [PubMed]

36. Schmidt, P.; Escobar, A.; Torres, J.; Martinez, C.; Rizzetti, D.; Kunz, S.; Vassallo, D.; Alonso, M.; Peçanha, F.; Wiggers, G. Aluminum exposure for one hour decreases vascular reactivity in conductance and resistance arteries in rats. Toxicol. Appl. Pharmacol. 2016, 15, 109-118. [CrossRef] [PubMed]

37. Srikanth, K.; Trindade, T.; Duarte, A.; Pereira, E. Cytotoxicity and oxidative stress responses of silica-coated iron oxide nanoparticles in CHSE-214 cells. Environ. Sci. Pollut. Res. Int. 2017, 24, 2055-2064. [CrossRef] [PubMed]

38. Peters, A. Ambient particulate matter and the risk for cardiovascular disease. Prog. Cardiovasc. Dis. 2011, 53, 327-333. [CrossRef] [PubMed]

39. Brook, R.; Sun, Z.; Brook, J.; Zhao, X.; Yan, J.; Mukherjee, B.; Rao, X.; Duan, F.; Sun, L.; Liang, R.; et al. Extreme air pollution conditions adversely affect blood pressure and insulin resistance: The air pollution and cardiometabolic disease study. Hypertension 2016, 67, 77-85. [CrossRef] [PubMed]

40. Mascarenhas, S.; Mutnuri, S.; Ganguly, A. Deleterious role of trace elements-Silica and lead in the development of chronic kidney disease. Chemosphere 2017, 177, 239-249. [CrossRef] [PubMed]

41. Grandjean, P. Even low-dose lead exposure is hazardous. Lancet 2010, 376, 855-856. [CrossRef]

42. Poręba, R.; Gać, P.; Poręba, M.; Andrzejak, R. Environmental and occupational exposure to lead as a potential risk factor for cardiovascular disease. Environ. Toxicol. Pharmacol. 2011, 31, 267-277. [CrossRef] [PubMed]

43. Tyrell, J.; Hafida, S.; Stemmer, P.; Adhami, A.; Leff, T. Lead (Pb) exposures promotes diabetes in obese rodents. J. Trace Elem. Med. Biol. 2017, 39, 221-226. [CrossRef] [PubMed]

44. Puett, R.; Hart, J.; Schwartz, J.; Hu, F.; Liese, A.; Laden, F. Are particulate matter exposures associated with risk of type 2 diabetes? Environ. Health Perspect. 2011, 119, 384-389. [CrossRef] [PubMed]

45. Coogan, P.; White, L.; Jerrett, M.; Brook, R.; Su, J.; Seto, E.; Burnett, R.; Palmer, J.; Rosenberg, L. Air pollution and incidence of hypertension and diabetes mellitus in black women living in Los Angeles. Circulation 2012, 125, 767-772. [CrossRef] [PubMed]

46. Song, C.; He, J.; Wu, L.; Jin, T.; Chen, X.; Li, R.; Ren, P.; Zhang, L.; Mao, H. Health burden attributable to ambient $\mathrm{PM}_{2.5}$ in China. Environ. Pollut. 2017, 223, 575-586. [CrossRef] [PubMed]

(C) 2017 by the authors. Licensee MDPI, Basel, Switzerland. This article is an open access article distributed under the terms and conditions of the Creative Commons Attribution (CC BY) license (http:/ / creativecommons.org/licenses/by/4.0/). 